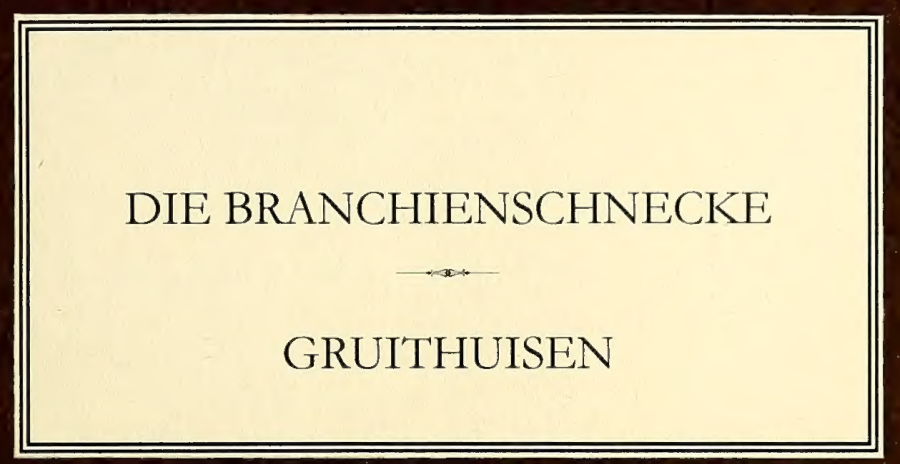




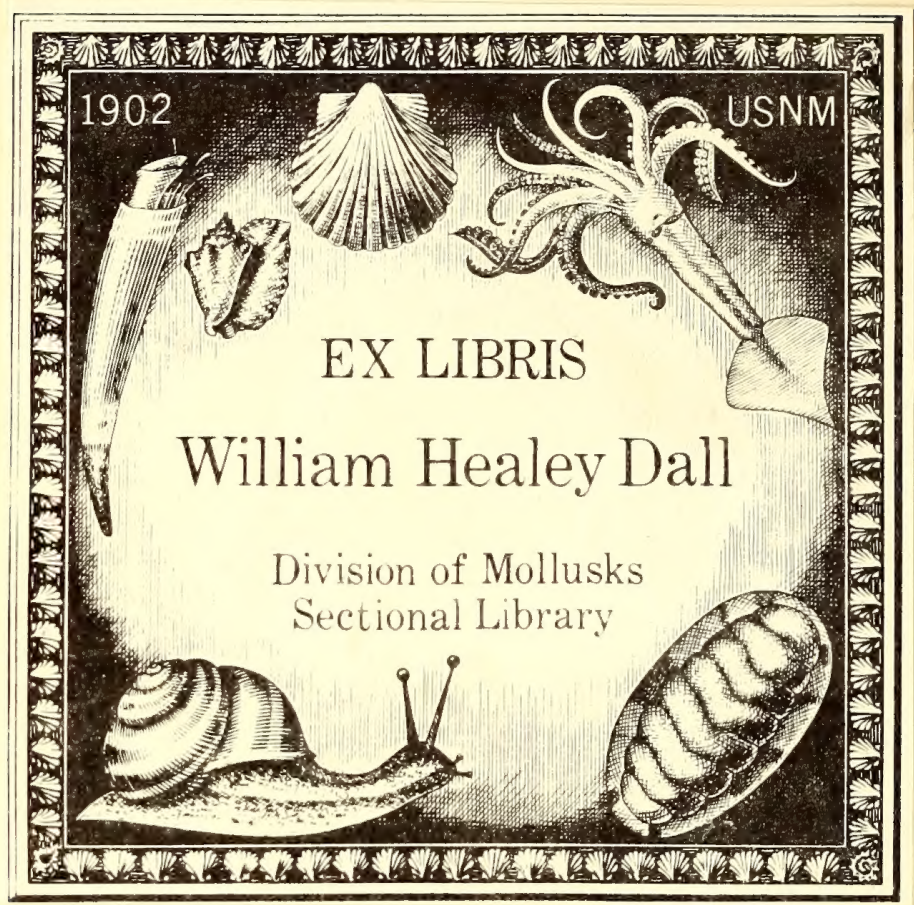






\title{
DIE BRANGHIENSCHNECKE
}

\author{
UND EINE.
}

AUS IHREN UEBERRESTEN HERVORWACHSENDE

\section{LEBENDIG - GEBAEHRENDE CONFERVE}

\author{
$\operatorname{VON}$ \\ Dr. F. v. P. GRUITHUISEN IN MÜNCHEN. \\ M. d. K. A. d. N.
}

Mit einer Kupfertafel. 
THSONIAN INSTITL

sill OกT $1+1949$

national museuH 

einen immerwährenden Wasserwirbel bewirkẹn. Die wie ein Ammonshorn gestaltete $S \mathrm{ch}$ ale hatei ne cirkelrunde Oeffnung, ist eine Linie hoch, und hat nur drei Windungen.

Wohnort: in stehenden Wässern, worin vielerlei Egel sind.

Geoffroy *) giebt eine Beschreibung von einer mit Federn gezierten Nerite, die, wie Müller ${ }^{\star *}$ ) und Schröter ${ }^{* * *}$ ) behaupten, mit der Valvata cristata Mïll. bei weitem keine Aehnlichkeit hat. Das von Müller unter dem. Namen Valvata cristata beschriebene Thier hat mit dem von mir bestimmten am meisten Aehnlichkeit. Allein so lange die sichtbar im Wasser einen Wirbel machenden Haare und das walzenförmige Organ an der rechten Seite des Kopfes von jenem nicht erwiesen sind, (was nur ein Naturforscher in der Gegend um Koppenhagen im Stande wäre, ) halte ich mich für berechtigt, die $V$. branchiata für verschieden von allen bisher beschriebenen federtragenden Schnecken zu halten,

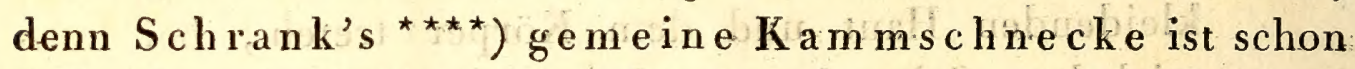
dadurch von der meinen different, dass deren Mund teller-

*) Von den Conchylien un Paris. Klasse I, Geschl, 1V. Gatt. I. S.102.

**) Miiller Verm. terrestr. et fluv. p. 198. Zoologiae danicae prodromus p. $\boldsymbol{X X X}$. et 2.39 .

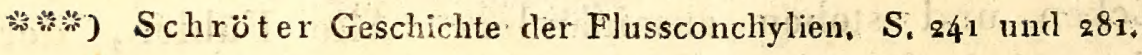

****) Fauna boicc. S. 262 und egz. Sammlung naturhistorischer Aufsäzo: S. 321. Tab, V. Fig. $9-12$. 
förmig, nicht gerüsselt, der hintere Theil des Fusses fast cirkelrund und das Ende des, dem Federbusch zur Seite stehenden Tentakels abgestumpft ist, andere Unähnlichkeiten zu geschweigen. Dr. Klees ${ }^{\star}$ ) hat unter dem Reichthum seiner Tübinger Testaceen drei Valvaten, aber keine federtragende; diese scheinen also im Ganzen ziemlich selten zu seyn.

Der Beweis, dass das federähnliche Organ, wie schon Greoffroy sehr einsichtsvoll behauptete, eine Art Kiemenfunction macht, ist leicht zu führen. I) Die Respiration der Infusorien wird einzig durch die Schwimmhaare befördert, wenn sie ruhen, absonderlich bei der Gattung Vorticella; und dieses ist noch immer der Fall bei schon höher stehenden Organisationen, die mit Mund und Darmkanal versehen sind, wie die Schrank'schen Planarien ${ }^{\star}$ ); weshalb wir auch berechtiget sind, zu sagen, dass die Bawegungshaare hier nicht mehr mit als Werkzeuge zur Beförderung der Einsaugung ${ }^{\star \star \star}$ ), sondern lediglich der Respiration dienen müssen. Dasselbe ist nun auch der Fall bei dieser Valvata, denn an diesem Thiere s'elean die Bewegungshaare so überall, dass sie sogar an der Sohle des Fusses nicht fehlen t). Dass aber da, wo Haut-

*) Dissert. inaug. zoologica, sist. characteristicen et descriptiones testaceorum, circa Tubingam indigenorum. Tub. 1818. Praes. Prof. Schübler. pag. 41 .

**) Salzburger med. chirurg. Zeitung. 1818. Bd. IV. S. 286.

**) Man vergl, meine Beiträge zur Physiognosie und Eautognosie. S. 112.

†) Ich glaubte, davon zum Theile das Gehen der Planarien und Schnecken ableiten zu können und besah sogleich unter starken K k k 
respiration im Wasser statt findet, Wasserwirbel, selbst bei allem Mangel an Bewegungshaaren, bemerkt werden, wenn innerhalb der Hant in Haarkanälchen rothes Blut mit Blutkügelchen *) circulirt, ist Sache der Erfahrung, wie das z. B. Anfangs bei den Kaulquappen der Frösche der Fall ist ${ }^{\star *}$ ), welches Phänomenal so nur durch elektrisch-chemische Attraktion und Repulsion zu Stande kommt. 2) Und endlich finden sich die zwei ungewöhnlichen Organe (das dritte Tentakel und der Federbusch,) genau an dem Orte, wo, wie wir wissen, viele Gasteropoden mit Schaalen, z. B. die der Gattungen Haliotis, Buccinum, Strombus, Murex u. a., die Respirationswerkzeuge haben, nur sind sie verborgen, wohl gar in eigene Höhlen versteckt, aber gar häufig zu Paaren vorhanden mit unähnlicher Ausbildung. Bei unserem Thiere ist also noch die ganze Vorrichtung zur Respiration nach aussen gekehrt, und nichts weiter.

Es ist sonderbar, dass ich an dem waIzenförmigen Organ, welches das Thier rechts am Kopfe trägt, keine Spur von Bewegungshaaren finden konnte. Dieses Organ hat duxchaus nicht das Vermögen, etwa wie die Fühler der Helix

Vergrössermugen des achromatischen Fraunhofer'schen Mikroskops die num bei mir in Mitte Decembers noch in den Gläsern vorhandenen Arten Buccinum auricula und Planorbis Vortex Mill. (vvelche nach $S$ chröter, S. 228, links gewunden ist, ) allein ich kann keine Spur von Bewegangshaaren orler Wasserwirbeln wahrnehmen.

*) Ich drücke mich darum so aus, weil einige $\mathrm{Naisarten,} \mathrm{T}$ ubifexe und Regenwürmer rothes Blut ohne Blutkügelchen haben.

5alzb, med, chir. Zeit, 1819. Bd. II. S, 447 . 
pomatia, sich zu verstecken, sondern es wird immer so entblösst getragen, wie es ist, ob es sich gleichwohl krümmen und nach allen Seiten neigen kann. Wenn nun dieses Organ, wie ich aus Analogie mit anderen Schnecken nicht zu zweifeln Ursache habe, das männliche Glied ist ${ }^{*}$ ), so ist bei diesem Thiere auch das männliche Geschlechtsorgan nach aussen gekehrt, und so verdiente dieser Schneck wohl eben so gut, als der Phallus impudicus, diesen Beinamen.

Wie und auf welche Art die Blutgefässe an den gewöhnlichen Fühlern hin- und herlaufen, eben so sieht man am ungewöhnlichen Fühler und am Federbusche Linien, wodurch der Weg dieser Gefässe, und vielleicht auch des Nervens, bezeichnet ist.

Die Empfindlichkeit ist bei dieser Valvata in allen Theilen gross. Sie zieht sich bei einer Erschütterung sogleich in ihre Wohnung und sperret die Hausthüre zu. Sie lebt aber auch unter einer grossen Menge von allerlei Ege!n, bei deren Verfolgungen eine so sperrbare Wohnung wohl eine gute Sache ist. Demungeachtet ist dieses Thier gar nicht häufig, ein Zeichen, dass es entweder nicht sehr fruchtbar seyn muss, oder dass nur wenige Eier, oder Junge, der Raubsucht der Egel entgehen. Am meisten Sensilität haben die Fortsäze des gefiederten Tentakels; denn wenn eine Cypris oder ein Cyclops eine Wasserbewegung erregt, so kräuseln sie sich, oder ziehen

*) Z. B. ganz genau an dieser Stelle ist die Oeffnung der Geschlechtstheile bei den Arten Limax u. a., nämlich unter dem oberen rechten Fühler. 
sich wohl sänmtlich fast gänzlich in das Innere zurück, ohné dass daran das Thier Theil nimmt. Auch einzelne. Strahlen ziehen sich zusammen, wenn ein Infusorium an eipe stösst; allein ich kann mich nicht erinnern, dass eine sich nach der gegenüberstehenden Seite gebogen hätte, wie es Schrank an seiner Valvata beobachtete, ob ich gleich glaube, dass die von mir beobachtete diese Kunst wohl auch zu üben versteht. Wir sehen hieraus, dass diese Organe der mit Schaalen bekleideten Gasteropoden zu den willkührlichen gehören. Geoffroy's Bestimmung: crista pennata, pennis undulatis, hingegen trifft hier nicht zu, denn im ungereizten Zustande sind die Seitenstrahlen nicht wellenförmig. Aber er spricht auch davon, dass das Thier in einem Glase Wasser herumschwimmt. Wenn diess keine allegorische Redensart ist, so hat seine Cochlea depressa cristata auch Ruderhaare. Frei schwimmen habe ich indessen meine Branchienschnecke nie sehen. Allein Geoffroy kann sie an der Oberfläche des. Wassers, oder an einem Schleimfaden, durch die Wassermasse haben kriechen sehen, was man aber wohl nicht schwimmen nenuen wird und was mehrere Wasserschnecken thun, besonders aus dem Geschlechte Buccinum. Davon wird er aher hoffentlich das Schwimmen nicht abgeleitet haben, wenn er hierauf sagt: ,, das Thier hebt dieses dritte Fühlhorn (nänlich neben dem Federbusch) in die freie Luft und bewegt es hin- und her;" denn zum Schwimmen würde es viel vortheilhafter den Federbusch gebrauchen. Dieses Organ ist bei weitem nicht so empfindlich, wie der Busch. Die Branchie ist überhaupt in ihrer ganzen Erscheinung gleichsam ein Thier für sich und einem Polyp sehr ähnlich: eben so scheu, ehen so empfindlich zusammenziehbar und eben so lüstern nach Sauerstoff, als der 
Polyp nacb Raub. Ich glaubte auch, als ich diese Valvata zum erstenmal erblickte, einen besonderen parasitischen Polyp in ihr entdeckt zu haben, nach der Analogie einer Abart der Nais diaphana, die in einigen Arten Buccinum wohnt, und sich genau so benimmt, wie diese Branchie.

\section{Confer $r$ a f e r a $x$}

Conferva. Die Samen sind ungleich gestaltet; die Pflanze ist ein harähnlicher Faden.

Conferva ferax; mit ganz einfachen, walzenförmigen, gelenklosen, gekammerten, zwei bis vier Linien langen Fäden, deren Kammern mit sich bewegenden Saamen angefüllt sind.

Wohnort: auf den todten, faulenden Ueberresten dex Branchienschnecke.

Ob ich gleich nicht gewiss weiss, dass diese Conferve bloss allein auf den Cadavern dieser Conchylienart wohnt, oder sich (wie die Pilze nur aus bestimmten organischen Resten wachsend) daraus nach Art der Infusorien generirt, (was am wahrscheinlichsten ist,) so habe ich sie doch nirgend anders gefunden, als da, wo sich das oben beschriebene Thier auf- 
hält und zwar in den grössten Bündeln immer nur aus den Oeffnungen, oder Lücken, ihrer Schaalen vorstehend. Zu zwei bis vier Fäden höchstens sah ich sie auf anderen kleinen Partikelchen, die ohne Zweifel aus der Schaale verloren giengen, am wahrscheinlichsten durch die Bisse der Egel.

Unter stärkeren Vergrösserungen des Mikroskops bemerkte ich in den Kammern eine gelblichbraune Masse. In der vordersten Kammer sah ich an lichteren Stellen, besonders vorn an der Spize, Kügelchen, die sich ste s sehr langsam bewegten und wälzten. Bei fortwährender Beobachtung zeigten sich folgende Phänomene : I) die Spize des Vordertheils einer Kammer dehnt sich in eine Biase aus; - 2) sie springt; - 3) aus ihr kommt innerhalb zivei bis fünf Minuten in schnellem Zuge nach einander ein Schwarm von durchscheinenden, brüunlichgelben Kügelchen zum Vorschein; - 4) diese Kügelchen schwimmen, gleich Infusorien, munter nach allen Richtungen herum; nach einigen Minuten aber ruhen sie aus und nur einzelne erheben sich und schwimmen eben so wieder eine Weile herum, und im ganzen Wasser, worin die Conferva sich befindet, kann man diese Körperchen antreffen und schwimmen sehen; - 5) innerhalb einer halben bis einer Stunde springt die Blase der zunächst nach rückwärts sich befindenden Kammer und die Körperchen kommen in die zunächst vordere Kammer, aus der sie aber sogleich durch ihre Oeffnung entfliehen, und so geht es, nur mit vermehrten Umsiänden, mit den übrigen Kammern, bis sie alle entleert sind;-6) haben die Körperchen in einer Kammer einmal mehr Raum, so tanzen sie nur in der Mitte derselben herum, gleichsam als öffneten sich Fächer, (die dem bewaffneten Auge entgehen,) 
in einen mittleren, gemeinschaftlichen Raum; - 7) am anderen Tage waren die Kammern des ganzen Bündels, welcher aus der (vergrössert abgebildeten) Schaale hervorstand, rein leer und - 8) in dem Wasser bewegte sich noch ein grosser Theil der, ihrer mütterlichen Hülle entflohenen, Körperchen herum.

Dringende Geschäfte hielten mich ab, zu sehen, was aus diesen Körperchen geworden ist. Ich glaube aber, dass, wenn sie keine Partikelchen von der todten Branchienschnecke getroffen hätten, ich weiter nichts, als ihren allmähligen Tod bemerkt haben würde.

Mehreremale brachte mich die Lebendigkeit dieser Körperchen auf den Gedanken, ob nicht mehrerlei Infusorien confervenartigen Gewächsen ihren Ursprung verdanken, und dass wir manche Confervensaamen in die Reihen der Infusorienarten einschwärzen. Indessen bemerken wir auch an veralteten Infusionen, dass darin Conferven und a uf denselben Byssus und Mucor entstehen ${ }^{*}$, und ich habe gesehen, dass in einer Nacht auf einer todten Capsularie, die sich unter dem achromatischen Mikroskop in einem Uhrglase mit Wasser befand, sich eine Art B y s s us entwickelt hatte. So nun bestätigt sich nur um so mehr, was ich längst aus eigener Erfahrung bahauptete ${ }^{\star \star}$ ): dass nämlich der Uebergang manches niedrigen Thieres in Pflanzenorganismen gewiss ist.

\footnotetext{
*) Meine Beiträge. S. 13\%。

\%) B eiträge, S, 32x, 322 .
} 
Aber eine gar grosse Frage ist: ob nicht einige Conferven selbst mehr Thiere als Pflanzen sind? Man bringe in ein breites Glas einen Büschel von $V$ a u cher's. Coniugata princeps *) und gebe ihr so wenig Wasser, dass die einzelnen Fäden kaum im Stande sind, sich gemächlich anszubreiten; dadurch wird ihre Begierde nach Sauerstoff sie zu stärkeren Bewegungen zwingen. Schon den anderen Tag wird man allerlei Wellen, Kegel und Hörner, von ihnen gebildet, auf der Oberfäche des Wassers erblicken und eine Menge davon wird an der inneren Fläche des Glases herauf gekrochen seyn und nach einigen Stunden wird man, bis sie gestorben, allemal beträchtliche Veränderungen wahrnehmen, welches acht Tage dauern kann.

Dieses und mehr andere thierische Phänomene, sogar eine eigene, ausserdem noch nirgend wahrgenommene Begattung, zeigen sich bei Vaucher's zweiter Confervenfamilie **). Ich habe zwei. Arten dieser Conferven untersucht und ich fand, dass sich diese gar sehr den Oscillatorien nähern. Am lebendigsten ist die Coniugata, welche Müller Conferva stellina nennt, woraus Vaucher mehrere Arten macht und die bei ihm Coniugata pectinata heisst ***). Auch habe ich bei

*) Ingenhousș (Versuche mit Pflanzén. Bc̣.III.S. 34.) hat die Erzeugung der Priestley'schen' griinen Materie aus den zerschnittenen infundirten Fäden dieser Conferva mit deı' Lebendigwerden der in ihr enthaltenen, in Spiralen gereihten Kügelchen verwechselt.

**) Vaucher Histoire des conferves d'eau douce. A Généve. 1803. 4io. S, 37. Conferves conjuguées.

*萧, Vaucher, p. 77. Tab. VII. fig. 4. 
dieser mit sehr starken Vergrösserungen durchs Mikroskop feine, schief abstehende Härchen wahrgenommen, womit wahrscheinlich die den Oscillatorien gleiche Bewegung, welche sie machen, vor sich geht. An der oben bereits angeführten Coniugata princeps Vauch. ${ }^{*}$ ) entdeckte ich Haare, welche noch feiner, als die der vorigen waren, wie gekörnt aussahen und von ihr in rechten Winkeln abstanden.

Freilich kann man bei solchen Conferven, welche (weil sie irgendwo festsizen, ) keine Ortsveränderung machen können, diese Art der Lebensäusserung nicht so leicht wahrnehmen; allein bei vielen zeigt sich doch das Leben des Saamens, wovon die Conf. ferax. das auffallendste Beispiel giebt, und welches Phänomen ich bei einigen Bacillarien ${ }^{* *}$ ), denen man doch die Thierheit gar nicht abspricht, wiedergefunden habe, z. B. bei Vibrio lunula Miill. ${ }^{* *}$ ), in deren beiden durchsichtigen Hörnern die Saamenkörnchen sich fortwährend bewegen $t$ ); dasselbe fand ich noch viel auffallender bei einer goldgelben, grossen, gemeinen, sehr lebhaften Bacillarie, die ich noch nicht determinirt habe, bei welcher die Körnchen im ganzen Leibe herumtanzen.

*) Ebend, p. 64. Tab, IV. fig. 1. Nach Müller Conferva ingalis, im Linne'schen System Conf, rivularis.

**) Müller (Animalcula infusoria. Havn. 1786. 4. p. 52 - 57. Tab. VII.) sezte, wie man aus dem Namen sieht, die Bacillarien sogar unter die Vibrionen, und Nitzsch (Beitrag zur Infusorienkunde, Halle 1817. S. 19.) fielen sie durch ihre willkührliche. Bewegung zum erstenuale auf.

**) Ebend, p, 55. Tab, VII, fig. 8 - 15 .

†) Meine Beiträge, S. 32z. Tab, II. fig. 40. 
Bei einigen Bacillarien kommt man sogar in Versuchung, sie für Stücke von Conferven zu halten *), denn was kann z. B. Aehnlicheres seyn, als Girod-Chantrans Confervenstück $K^{\prime \prime}$ fig. 77 der 33 . Tábelle **) mit einem Exemplar der Vibrio lunula Müllers. Andererseits kommt man in nicht geringe Verlegenheit, wenn man ein kleines Stück einer Oscillatorie daher wandern sieht, und nur eine geringe mikroskopische Vergrösserung hat, zu bestimmen, ob es zu den Bacillarien, oder Conferven, oder Oscillatorien gehöre ****).

Aehnliche gekammerte, saamengebährende Conferven, welche aber auf vegetabilischem Grunde vegetiren, trifft man in Girod-Chantrans Werk mehrere an $t$ ), allein mit einer so excessiven Fruchtbarkeit nicht; und ich habe solches nur noch bei einem Thiere gefunden, welches zwischen Vibrio und Gordius mitten inne steht.

*) Nichts davon zu sagen, dass man wahre Bacillarien für Conferven hielt, wie z. B. die Miillersche Conferva pectinalis. Ich theile also Nitzsch's Meinung (ebend. S. 110.) nicht, seine Bacillaria pectinalis fuir verwandt mit den Conferven zu halten; denn sie ist ein Band und kein Faden, wenn sie noch nicht zerfallen ist.

**) Girod-Chantran Récherches chim. et microscopigues sur les conferves, Bisses, Tremielles, etc. A Paris 1802. p. 227.

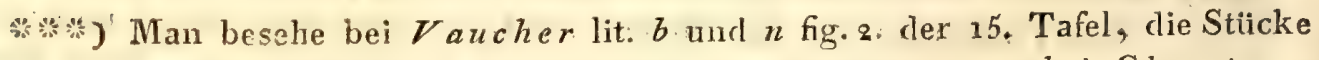
der Oscillatoria princeps, und den Bisse de cave bei Chantran (Tab. XXVIII. fig. 68. pag. 195.), welcher lebt und., von Vaucher p. 153 für eine Oscillatoria erklärt wird, aber aus lauter Bläschen zusammengereiht ist.

†) Vergl, 1nr, 44, 71, 77 und die eben so bezeichneten Figuren. 
E r k l a r u g d e r F i g u r e n.

Tab. XXXVIII.

Fig. 1. Die Branchienschnecke in ihrer natürlichen Grösse.

Fig. 2. Durch das achromatische Mikroskop vergrössert.

Fig. 5. Durch ein scharfes Suchglas von vorn angesehen, wobei sie bei $a$ die Branchie, bei $b$ den ihr entsprechenden Fühler, bei $c c$ die ächten Fühler, bei $d$ das männliche Glied, bei $e$ den Rüssel, bei $f$ die Augen (welche man in der Hauptfigur wegen der Tentakeln nicht sieht,) und bei $g$ die vorderen Enden des Fusses zeigt.

Fig. 4. Die Durchmesser der Windungen, nach einem senkrechten Durchschnitte der Schaale in der zweiten Figur; um zu zeigen, dass die Kreise darin cirkelrund, nicht in einander gedrückt, und dass die Windungen, wie beim Ammonit, aufgerollt erscheinen.

Fig. 5. Das oberste Stück der Branchie (wie die folgenden Figuren unter starker Vergrösserung) mit ihren Mittelstreifen für Gefässe und Nerven. Da hier die Gefässe nur Haarkaräle sind, so glaube ich, dass sie sich zwischen dem äusceren Rande und den Streifen, - die Nerven aber innerhals der Streifen befinden. Es ist die ganze Aussenfläche des Busches mit Härchen versehen, welche sich nnaufhörlich bewegen.

Fig. 6. Das oberste Stück des Fühlers neben der Branchie, weIcher eigentlich die andere, einfache Branchie ist. Devon ist das obere Ende mit einer stumpfen Spize und der Fühler ist auf seiner ganzen Oberlläche mit ähnlichen Bewegungshaaren versehen; auch sieht man die Streifen des Verlaufes der Nerven und Gefässe.

Fig. 7. Das oberste Stück eines am Kopfe sizenden Fühlers, welcher oben abgestumpft erscheint, und eben so mit Bewegungshaaren und Streifen versehen ist.

Fig. 8. Das Hinterende des Fusses, worauf der Deckel befestigt ist, welcher zum Verschliessen der-Sehaale gehört. Die Darstellung von oben. 
Fig. 9. Eines der vorderen Enden des Fusses. Jenes (Fig. 8.) und dieses ist, so wie die ganze übrige Fläche des Fusses, mit Bewegungshaaren besezt.

Fig. 10. Der Deckel, von der äusseren, concaven Seite betrachtet, um darin seine concentrischen Streifen zu zeigen, Die innere Seite ist convex (s. Fig. 2.)

Fig. 11. Das Mundende des Rüssels, gleichfalls vorn um und um mit Bewegungshaaren besezt.

Fig. 12. Der obere Theil der Branchie, um zu zeigen, wie sie sich in allen Theilen kräuselt, wenn sie sich, wegen Reizung, verbergen will.

Fig. 13. Eine durch das Mikroskop vergrösserte Schaale der Branchienschnecke. a a a. Conferven, an welchen man die vorderen Kammern schon leer erblickt; - bb. an welchen sie noch voll sind. ( $c c$. Vorticellen, die sich an dem vermoderten Eingeweide der Schnecke, welches durch die zerbrochene Schaale bloss gelegt ist, angesezt haben.)

Fig. 14. Eine vergrösserte Conferve, in deren erster Kammer a die lebendigen Saamen reif und deutlich, in der zweiten Kammer $b$ aber minder - und in der hintersten $c$ am wenigsten deutlich erscheinen. Bei $d$ und $e$ bilden die Fächer der Kammern helle Zwischenräume.

Fig. 15. Das Vorderende einer solchen Conferve (unter sehr starker Vergrösserung), wie sie bei $a$ eben ihre lebendigen, mứnter herum schwi:nmenden Saamen gebährt, $b$ Das Vorderende der zweiten Kammer: die Wasserblase ist bereits gespannt und zum Springen bereit. Diese Blase scheint von der hintersten Wand der vorderen Kammer gebildet zu werden und entsteht, sobald der Druck von den vor ihr liegenden Saamen nachlässt.

Fig. 16. Eine Conferve, unter der Vergrösserung von Fig. 14. a. b. c. stellen drei ganz leere Kammern dar, worin nicht ein einziger Saame zurück geblieben ist. 

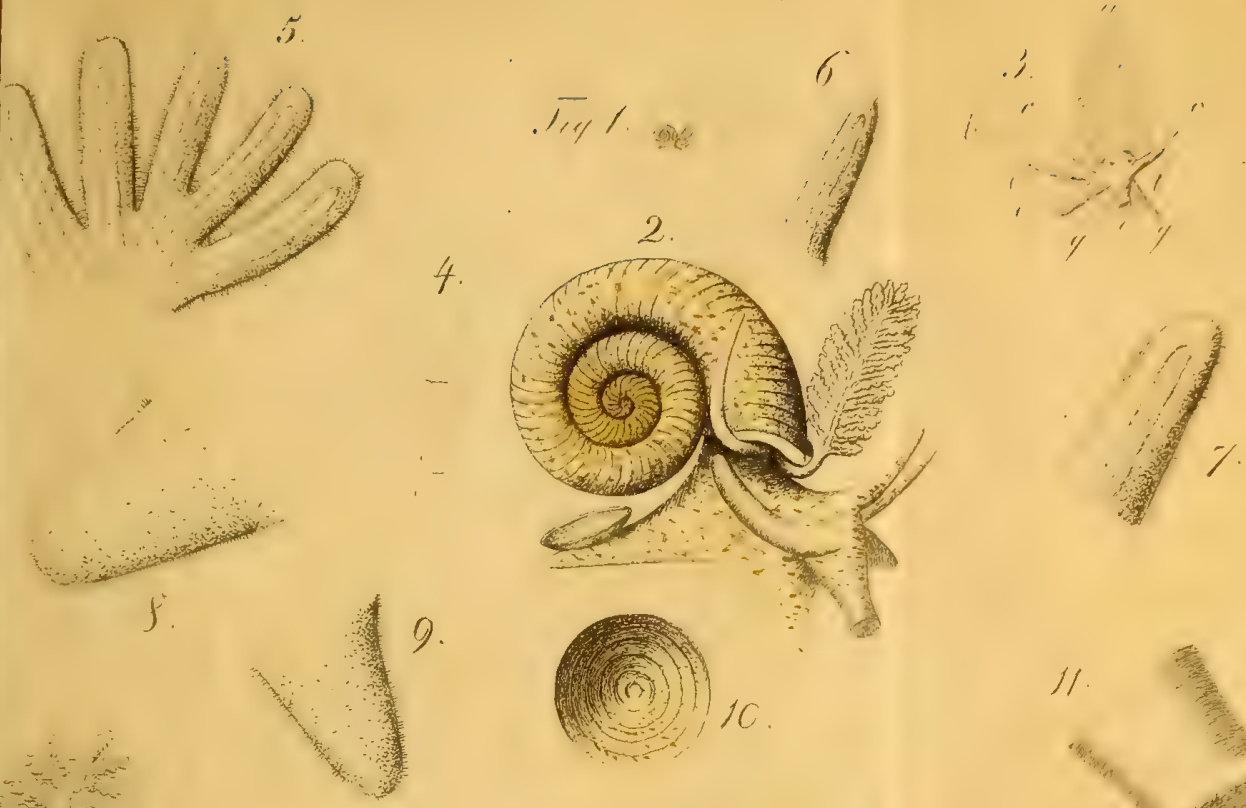

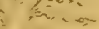

$\because \quad \cdots 12$

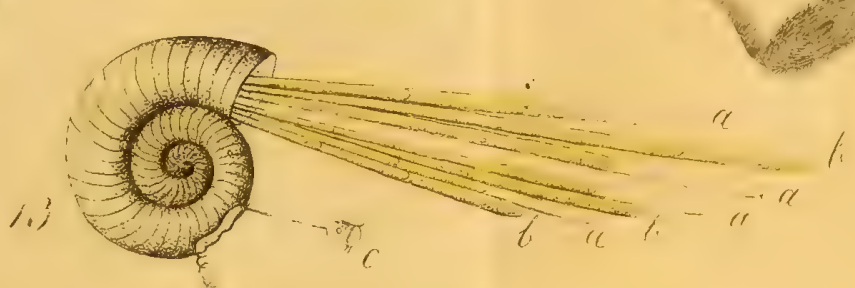

te $r$
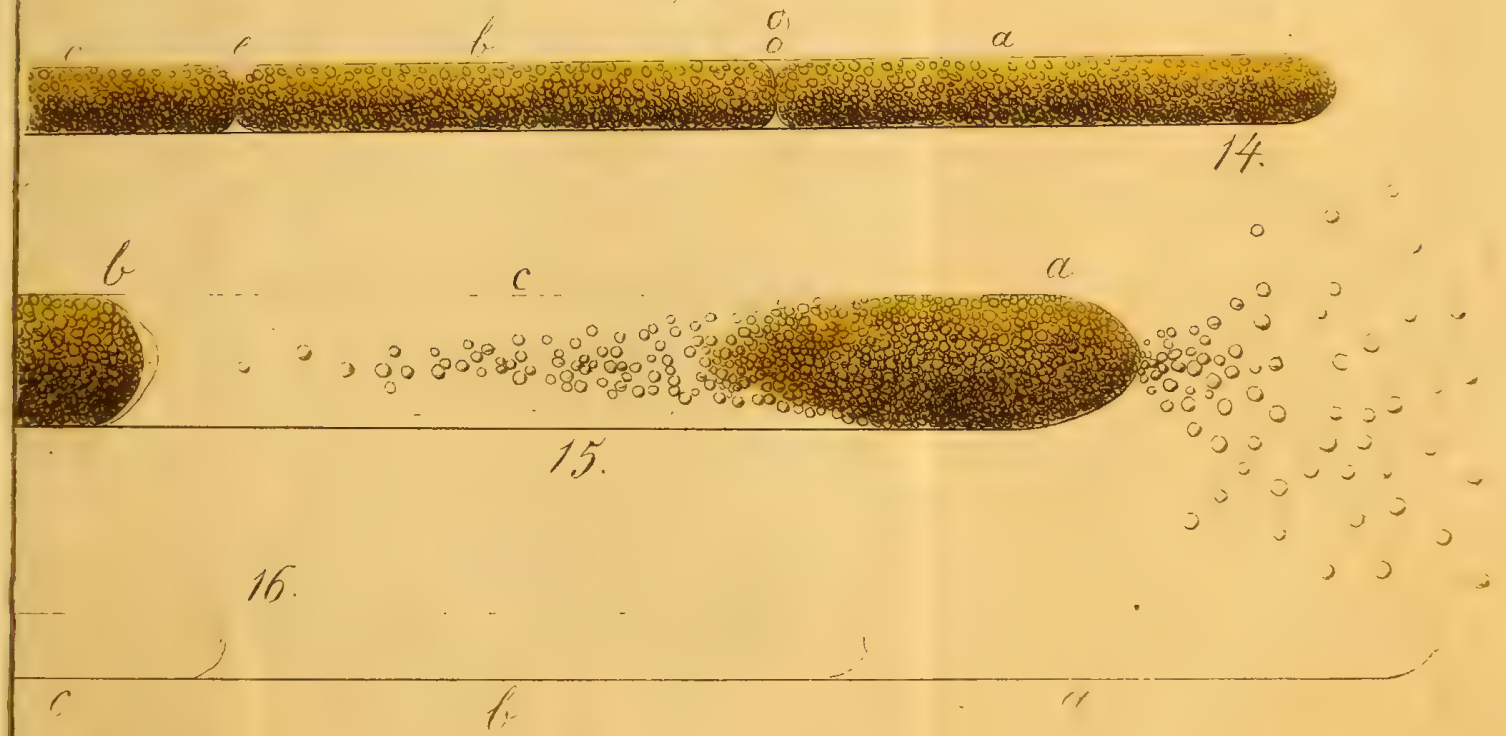
2.
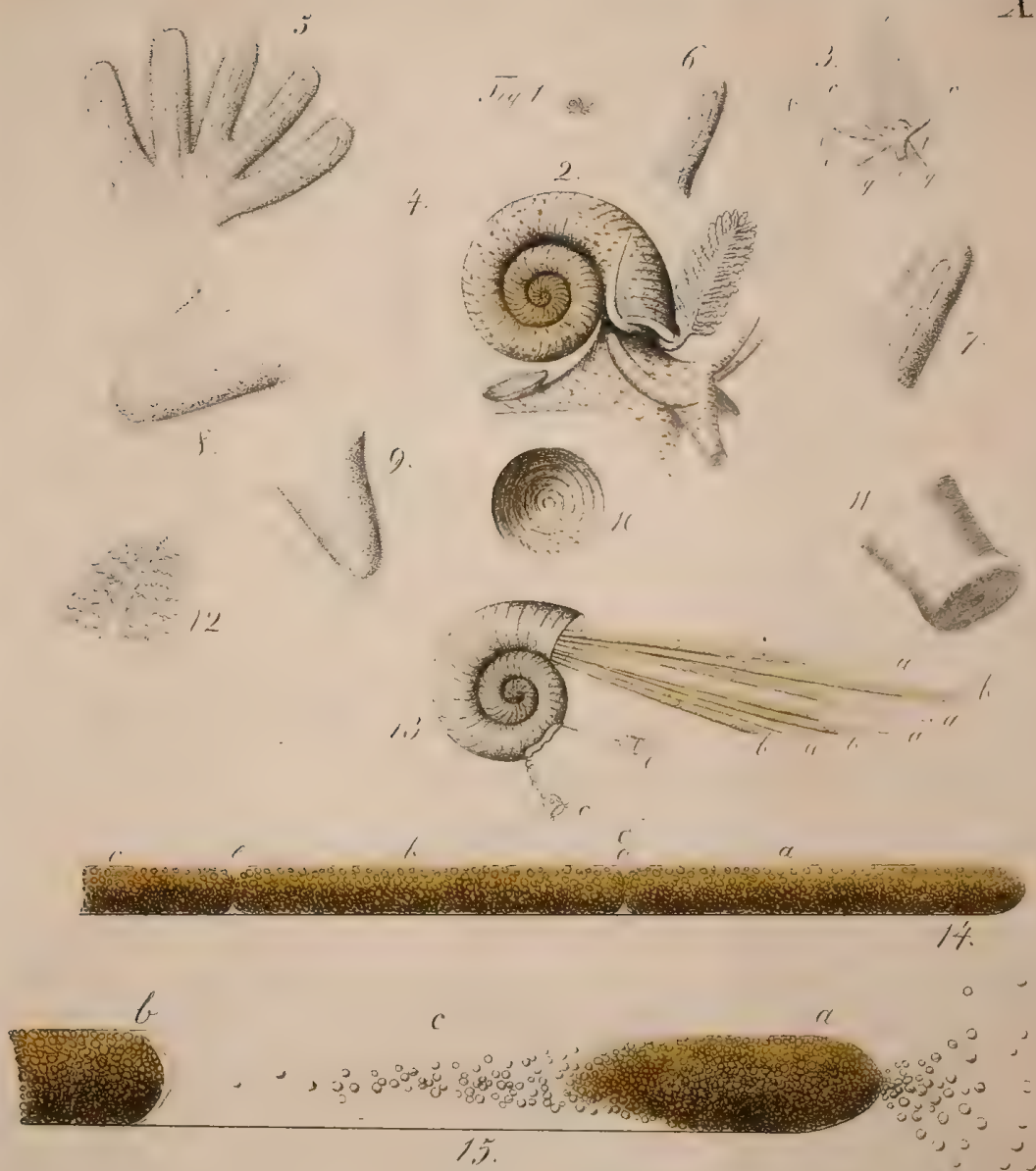

16
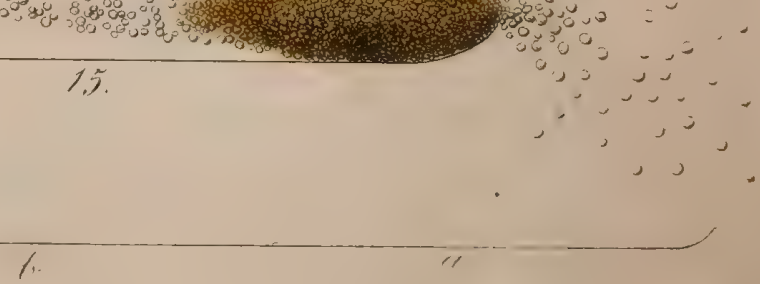

lett. it 



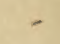



